\title{
Effects of high-intensity interval training associated to microcurrent on localized adiposity: protocol of randomized clinical trial
}

\author{
Aline Pesaski ${ }^{1}$, Nandara Lopes dos Santos ${ }^{1}$, Natalia de Paiva da Silva ${ }^{1}$, Rubia Alana Souza dos Anjos ${ }^{1}$, Audrin Said \\ Vojciechowski ${ }^{2}$, Ana Carolina Brandt de Macedo ${ }^{3}$, Rubneide Barreto Silva Gallo ${ }^{3}$, Raciele Ivandra Guarda Korelo ${ }^{*}$.
}

1 Universidade Federal do Paraná (UFPR), Curitiba, (PR), Brasil; 2Programa de Pós-Graduação em Educação Física, Universidade Federal do Paraná (UFPR), Curitiba, (PR), Brasil; ${ }^{3}$ Departamento de Prevenção e Reabilitação, Universidade Federal do Paraná (UFPR), Curitiba (PR), Brasil.

\begin{abstract}
Background: High-intensity interval training promotes body weight loss, while microcurrent electrical stimulation has therapeutic potential to reduce localized abdominal fat. However, there are no studies that have investigated the association of the two forms of intervention in reducing localized adiposity. Objectives: To verify the effects of high-intensity interval training, associated or not, with microcurrent therapy, in reducing localized abdominal fat. Methods: A randomized controlled clinical study will be conducted with 60 women aged 18 to 40 years, with localized abdominal adiposity. Participants will be randomized into 3 groups: Control Group (without physiotherapeutic intervention), Exercise Group (high-intensity interval training for 30 minutes) and Exercise Group associated with Microcurrent (application of 30 minutes of microcurrent prior to high-intensity interval training). The intervention will take place twice a week for 5 weeks. The clinical outcomes evaluated and their respective measuring instruments will be: body composition (bioimpedance scale and adipometry), anthropometric measurements (perimetry), level of physical activity (International Physical Activity Questionnaire - IPAQ, short version), quality of life (IWQol-Lite, short version), body satisfaction (Stunkard figure rating scale), degree of satisfaction with the performance of the intervention (questionnaire adapted by the researchers) and evaluation of the lumbopelvic complex. These outcomes will be measured in 4 moments: before the intervention, after the 5th and 10th intervention, and with a follow-up of 1 month. Discussion: A previous study has already shown the positive result of the association of moderate intensity exercise, associated with the previous application of microcurrent, in reducing localized abdominal adiposity. Thes e findings raised the hypothesis that the association of this electrical therapy with high-intensity interval training may present positive results in the search for the reduction of abdominal fat, with the clinical advantage of enhancing the achieved results and/or reducing the time spent by the patient in therapy. Keywords: Body Composition; High Intensity Intermittent Exercise; Electric Stimulation Therapy; Physiotherapy.
\end{abstract}

\section{BACKGROUND}

The accumulation of localized adipocytes in the abdominal wall, or localized lipodystrophy, is a major concern with body aesthetics ${ }^{(1)}$. In fact, it is already established that this accumulation increases the lipolytic activity in the subcutaneous and visceral adipose tissue, causing an increase in free fatty acids in the blood circulation and in the hepatic triglyceride synthesis, causing the appearance of the metabolic syndrome and, consequently, increasing the risk of cardiovascular diseases $^{(2)}$. Additionally, adipose tissue plays an important role in developing of a systemic inflammatory state, through the secretion of cytokines and chemokines, predisposing the individual to numerous other inflammatory diseases ${ }^{(3)}$ and promoting functional physical limitations ${ }^{(4)}$. Allied to these factors, the sedentary lifestyle and the high calorie and high-fat diet, adopted by a large portion of the population, contribute negatively to the worsening of health status and the accumulation of abdominal localized adiposity ${ }^{(5)}$. In the midst of this scenario, the search for methods to reduce body fat is constant, and the practice of physical exercises is one of the most used(6). Published guidelines for your practice $^{(7)}$ generally recommend aerobic exercises of high volume (150 minutes/week) and moderate intensity (55$69 \%$ of heart rate $(\mathrm{HR})$ max/maximum heart rate or $12-13$ on the Subjective Perceived Exertion scale (SPE); however few people are able to comply with these recommendations, with lack of time being one of the main reasons for engagement ${ }^{(8)}$.
Thinking of a time efficiency strategy, it is suggested that high-intensity physical training (70 to $89 \%$ of $\mathrm{HRmax}$ or 14-16 in SPE) for 20 minutes on 3 days of the week, also has the potential to promote the reduction of abdominal adiposity ${ }^{(7)}$. Thus, High-Intensity Interval Training (HIIT) stands out, defined as an intermittent period of effort performed above $80 \%$ of HRmax, interspersed with recovery periods ${ }^{(9)}$. Meta-analysis ${ }^{(10)}$ published in 2019 proved that training with HIIT reduced $28.5 \%$ of the total absolute fat mass, in comparison to the high volume exercise, besides being practiced in less time $^{(11)}$ and provide high adherence rates for its practice in overweight and obese adults ${ }^{(8)}$.

But in fact, body contouring surgical techniques have become the gold standard for removing subcutaneous adipose tissue, as they promote immediate results. However, they have inherent risks and complications such as: pain, edema, hematoma and scarring, in addition to prolonged recovery ${ }^{(12)}$. Thus, numerous noninvasive lipolytic techniques alternative to liposuction have been developed in the last decades, including: cryolipolysis, focused ultrasound, low intensity laser therapy, radio frequency and local applications of lipolytic injections ${ }^{(13-14)}$. These techniques are less painful or even painless, but they also have minimal inherent risks. The expected clinical results with the application of these resources aim at lipolysis (enzymatic breakdown of triglycerides in glycerol and free fatty acids) permanently, consequence of the death of adipocytes ${ }^{(15)}$. 
In contrast, electrical currents applied transcutaneously, such as microcurrent, can be used in order to promote temporary electro-lipolysis (justified by a neurohormonal mechanism due to the stimulation of catecholamines that activate lipases and allow the hydrolysis of triglycerides), increase metabolism, increase the local blood flow and promote lymphatic drainage $^{(16)}$. It is suggested that with electrolipophoresis, the fatty acids and glycerol released into the bloodstream become the main energy source during physical exercise ${ }^{(17)}$. Currently, interest in this current is growing as its effects occur at the cellular level (normalizing bioelectricity) and its application occurs in subsensorial microamperage, i.e., it is not associated with the uncomfortable sensation as in other therapeutic currents, being painless ${ }^{(18)}$. In addition to not having side effects, being low cost and easy to apply ${ }^{(19)}$. Due to the scarcity of studies on the application of microcurrent electrolipolysis, it is difficult to establish an adequate protocol and implies uncertainties as to the electrical parameters that should actually be used. Thus, to achieve these therapeutic benefits, dosimetry of the resource becomes a crucial point and involves regulating the following parameters: stimulation frequency, current amplitude, electrode position, duration of application, number of applications per day and number of days a week ${ }^{(19)}$. Regarding the association between microcurrent and physical exercise, it was evidenced that the transcutaneous application of microcurrent followed by aerobic exercise of moderate intensity, promote the reduction of abdominal and visceral fat in overweight ${ }^{(5,20-21)}$ and cardiac patients $^{(2)}$. However, the prior application of the microcurrent associated with HIIT is still unknown. Therefore, the possible advantage of this association would be to promote a cumulative effect in the mobilization of the adipose panicle, allowing its bioavailability as an energy source, in a protocol of shorter physical exercise time, providing means for the treatment of localized fat. Thus, the present protocol of randomized clinical trial has as main objective to evaluate the effects of HIIT associated or not with the previous application of microcurrent therapy in the reduction of abdominal fat in adult and sedentary women. The hypothesis of this study is that HIIT performed twice a week when combined with microcurrent for 5 weeks is sufficient to reduce abdominal localized adiposity and its related body measurements. In addition, it can improve quality of life, body satisfaction and functionality of the lumbopelvic complex muscles.

\section{METHODS}

\section{Type of study}

This study is a randomized controlled clinical trial. The study will be conducted at Universidade Federal do Paraná (UFPR) and was approved by the Research Ethics Committee of Departamento de Ciências da Saúde da Universidade Federal do Paraná (CAAE: 55963816.2.0000.0102/ Approval number:1.689.345) following the recommendation of the Standard Protocol
Items: Recommendations for Interventions Trials (SPIRIT) and the results of the clinical trial will follow the CONSORT guidelines ${ }^{(22)}$. All items of the WHO Trial Registration Data Set were registered in public access in the Brazilian Registry of Clinical Trials (RBR96sw76).

\section{Eligibility criteria for participants}

Participants will be recruited through social networks, local newspapers and radio advertisements. The subjects will be guided in relation to the research proposal and treatment protocol, and those who are interested in participating will be selected according to the inclusion and exclusion criteria. Women residing in Curitiba (PR) and region will be invited to participate in the study.

\section{Inclusion criteria}

The inclusion criteria will be: Sedentary women, aged between 18 and 40 years and with the presence of abdominal fat (thickness of adipose subcutaneous tissue of the abdominal wall) greater than $15 \mathrm{~mm}^{(23)}$ confirmed by adipometry, using as a reference point 2 $\mathrm{cm}$ from the right side of the umbilical scar ${ }^{(24)}$. The disclosure will be made by a digital pamphlet published on social networks and in printed form spread over Universidade Federal do Paraná (UFPR). Associated waist circumference and localized abdominal adiposity are related to one of the causes of mortality in the adult population. In addition, about $31 \%$ of adults are insufficiently active worldwide ${ }^{(25)}$, and HIIT can be applied to increase adherence to exercise. The decision to evaluate only women was mainly since they have greater abdominal adiposity than men, justifying interventions for this population. Those interested will have an appointment time for evaluation by completing a structured and applied evaluation form with the purpose of collecting sociodemographic and clinical data, as well as, detecting the presence of exclusion criteria through interviews, and physical evaluation with anthropometric measurements collection.

\section{Exclusion criteria}

The participant must not be on a diet; have smoking and drinking habits; make use of weight loss medications, corticosteroids, progesterone and diuretics; be pregnant or postpartum less than one year; have electronic monitoring or metallic implants in the pelvic region; in dermatofunctional treatment for the abdominal region; performed surgery and/or radiotherapy from the abdominal/pelvic region less than 6 months ago; carriers of diseases of the lymphatic system, cardiovascular system, thrombophlebitis, acute infection, diseases of the central nervous system, tumors and diabetes.

\section{Sample Calculation}

The sample calculation was performed based on the lean mass assessed by bioimpedance. A previous study ${ }^{(2)}$ found an increase in lean mass in female participants (18-30 years) after applying microcurrents 
(transcutaneous, frequency between 10 to $25 \mathrm{~Hz}$, maximum tolerable intensity, 30 minutes) and then a cycle ergometer (moderate intensity) for 30 minutes. The mean intragroup difference was $-1.1 \mathrm{~kg}$ in the placebo group and $0.7 \mathrm{~kg}$ in the intervention group, resulting in an effect size of 0.2 (small effect). In this study, the sample size was calculated using the $G^{*}$ Power 3.1.9 program, with an effect size of 0.2 ; significance level of 0.05 (type I error); and 0.80 sample power. Therefore, 54 participants will be needed, with 18 in each group. However, considering possible sample losses and aiming to increase the effect size, $10 \%$ of the total will be increased, resulting in 60 participants, 20 in each group.

\section{Recruitment}

Interested participants should contact one of the research members, receiving information about the project and will then be invited to schedule an initial assessment. Before the evaluation, they will receive more detailed explanations of voluntary participation in the research, with the removal of doubts and if they agree, they must sign the Free and Informed Consent Term that was reviewed and approved by the Research Ethics Committee, as well as, the Image Use Term, with two copies of each (one copy remaining for the research and the other with the participant). The collected data will be in safe places, identified only by the participants' codes and will be used for publication in scientific journals without any identification. At the end of the study, each volunteer will receive a report with the evaluations performed and those who have not received any type of intervention will have the opportunity to decide whether they want to try any application.

\section{Randomization and allocation concealment}

After completing the pre-intervention assessments, the participants will be randomized into 3 groups: CG (control group), EG (exercise group) and MEG (microcurrent and exercise group). Randomization will be performed in blocks ${ }^{(26)}$ with 20 participants in each group. Participants will be informed after randomization about the type of intervention they will receive. The researcher who will be responsible for the evaluations will be blinded to the allocation and intervention of the groups to not interfere indirectly in the results. Due to the types of intervention, participants will know the group they were allocated to. The study design and participants' schedule are shown in figure 1.

\section{Interventions}

After randomization, participants will be allocated to one of three study groups: Control Group (CG), without physical therapy intervention, Exercise Group (EG), high-intensity interval training and Microcurrent and Exercise Group (MEG), application of the microcurrent previously to the application of high-intensity interval training. The interventions will occur for 5 weeks, with a frequency of 2 times a week, on the same day and time, with a minimum interval of 48 hours, totaling 10 applications, with varying duration depending on the group's allocation (EG: 30min, MEG: 60min). Physical training and the application of the current will be offered free of charge to participants. All participants will be instructed to continue with their eating routine normally and not to practice any other type of physical exercise and/or any method for reducing fat.

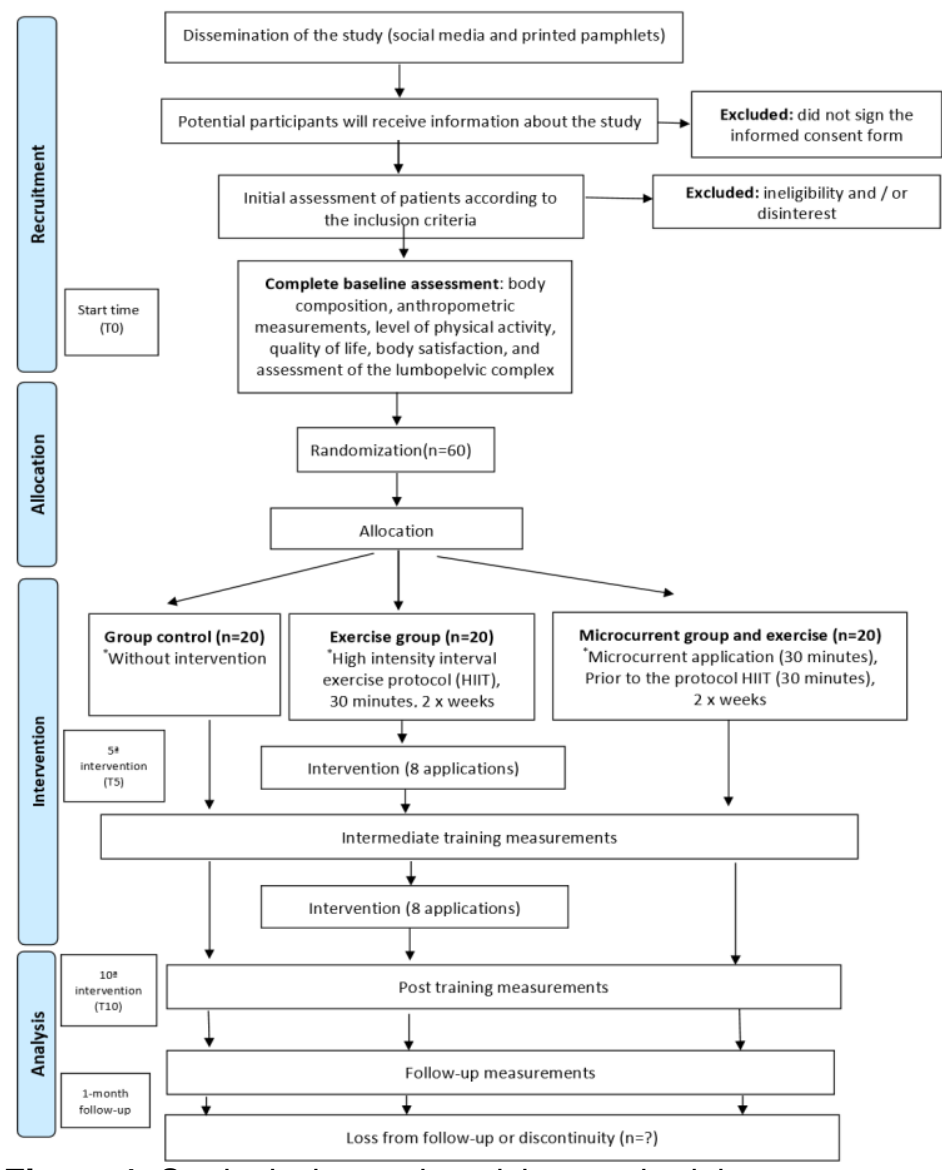

Figure 1. Study design and participant schedule.

\section{Control Group}

The CG participants will not receive any application during the 5-week period, only the four evaluation moments. They will be instructed not to change their usual activities during these weeks. After the trial period, intervention will be offered at no cost to those who are interested.

\section{Exercise Group}

A standardized physical exercise protocol with a total duration of 30 minutes will be used, supervised full time by the researcher. The protocol will be divided into three parts: 1) initial 5 minutes of warm-up on the exercise bike(27); 2) 20 minutes of a high-intensity interval training circuit containing 10 sets of 60 seconds ${ }^{(28)}$ interspersed with active rest of 60 seconds; 3) 5 minutes of cooling down ${ }^{(27)}$. An additional file shows this in more detail [see Additional file 1]. The intensity of the exercise will be monitored during the performance of the protocol by the researcher and participant previously oriented with the use of Polar frequency meter (above $80 \%$ of the HRmax) and Borg's Rating (6- 
20) of Perceived Exertion Scale (29) (above 15-tiresome). The maximum $\mathrm{HR}$ calculation ${ }^{(30)}$ will be performed using the $\mathrm{HR}_{\text {theoretical maximum }}=208-0.7 \times$ age. From this calculation, together with the measurement of resting $\mathrm{HR}$, the training $\mathrm{HR}$ corresponding to an intensity equal to $80 \%$ will be calculated using the Karvonen's formula ${ }^{(7)}, \mathrm{HR}_{\text {training }}=\mathrm{HR}_{\text {rest }}+\%$ intensity $\times\left(\mathrm{HR}_{\text {maximum }}-\right.$

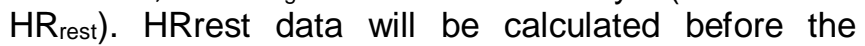
application of the exercise protocol and after the data has been collected and the values obtained in the 12th and 22nd minutes will be recorded in the physical exercise monitoring form of each participant.

\section{Microcurrent associated with Exercise Group}

The group will be submitted to the application of microcurrent prior to the performance of the exercises. Neurodyn Esthetic, Ibramed equipment will be used, in which the microcurrent is defined as a rectangular pulsed single-phase current with automatic polarity inversion every 3 seconds and operates at a maximum amplitude of 500 microamperes $(\mu \mathrm{A})$. The following parameters will be used ${ }^{(5,20-21)}$ : frequency of $25 \mathrm{~Hz}$ in the first 15 minutes, changing to $10 \mathrm{~Hz}$ in the next 15 minutes, with maximum intensity $(500 \mu \mathrm{A})$ or subsensory level. For the application of the therapeutic electric current, with the patient in supine position (figure 2), four carbon-silicon electrodes (50 $\mathrm{mm}$ width $\mathrm{x}$ $25 \mathrm{~cm}$ ) coupled with electroconductive gel, will be used positioned in a vertical bipolar arrangement after asepsis with alcohol, on the abdominal fat accumulation region, and, fixed with Velcro band as follows: for the first channel, an electrode will be positioned $2 \mathrm{~cm}$ to the right of the upper border of the umbilical scar and another $2 \mathrm{~cm}$ to the left; while for the second channel the two electrodes will be positioned $50 \mathrm{~mm}$ below the two electrodes of the upper channel. After applying the current, the group will perform physical training following the same exercise protocol used in the EG.

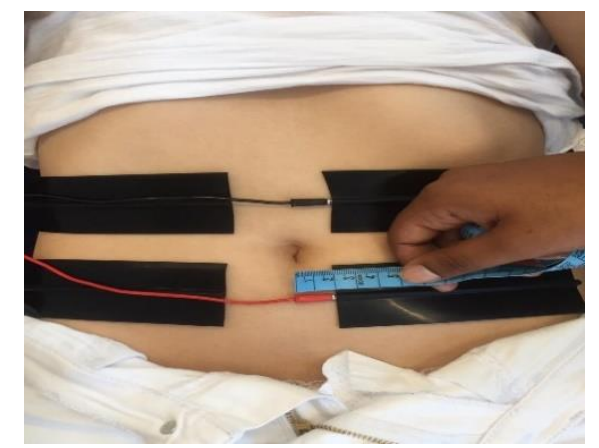

Figure 2. Positioning of electrodes for Microcurrent application.

*Note: for the possibility of visualizing the positioning of the electrodes in the figure, they were not fixed with a velcro band, according to the method described for application.

\section{Discontinuation of treatment}

The HR will be measured before, during and after the performance of the exercises by a polar heart-rate monitor, so that the participant reaches the proposed training range. In the event of any complications the training will be suspended. Previously to the current applications, participants will be guided on the sensation during the application, being able to communicate any discomfort; there will be an inspection of the skin in the region before the procedure and after it is done; the use is suspended if there is any complication. Participants who do not comply with the entire assessment and intervention protocol, who initiate any other treatment program for localized adiposity or who request their exclusion, will be discontinued from the study.

\section{Clinical Outcomes}

The following clinical outcomes will be used to measure the results, measured in 4 moments: before the intervention, after the 5th and 10th intervention, and with a follow-up after 1 month.

\section{Primary Outcomes}

1) Body composition: will be verified by 2 different methods (bioimpedance and adipometry). Bioimpedance will be performed with the electric scale (HBF 214, Omron®) to check total body mass, body mass index, body fat percentage, visceral fat percentage, muscle mass percentage, body age and basal metabolism. For its performance, the participant will be instructed to wear a top / shorts or bikini; do not drink alcohol, coffee, energy and carbonated drinks in the last 24 hours before the evaluation; avoid consumption of fried foods and sweets in the last 24 hours; do not perform vigorous exercise for 24 hours, empty your bladder half an hour before measurement ${ }^{(21)}$ and also, avoid the consumption of food and drinks until 4 hours before the test is performed and not be in the menstrual period(31). Adipometry will check five skin folds (tricipital, mid-axillary, suprailiac, abdominal and thigh), using a scientific adipometer (Top TecCescorf $(\AA)$, in the right hemidium, with the participant in an orthostatic and relaxed position. Each fold will be measured three times and the arithmetic mean will be used for statistical analysis. After measurement, the body fat percentage, fat weight and lean weight will be estimated, as proposed by Jackson and Pollok ${ }^{(24)}$ and Faulkner(32).

2) Anthropometric measurements: abdominal perimetry will be performed using a tape measure, with the participant in the orthostatic position, measured after expiration, in four points ${ }^{(20)}$ : a) waist - narrowest point between the last rib and iliac crests, b) greater abdominal circumference, c) hip - under the trochanters of the femur and d) immediately above the iliac crests. Based on these measurements, the Waist-Hip Ratio (WHR) will be determined, obtained by dividing the waist circumference by the hip ${ }^{(33)}$. Body height will also be checked to calculate the Body Mass Index.

\section{Secondary Outcomes}

1) Physical activity level: measured by the International Physical Activity Questionnaire (IPAQ), short version, validated in Brazil ${ }^{(34)}$. The instrument assesses the intensity of physical activity in three 
domains (walking, moderate activity and vigorous activity) and the time spent performing them in the week preceding the assessment, in addition to including a record of the time the subject remains seated. The results obtained will be calculated ${ }^{(35)}$ in minutes per week and the subjects will be classified according to the frequency and duration criteria in: sedentary, insufficiently active, active and very active.

2) Weight effects on quality of life: it will be used the IWQOL-Lite questionnaire, short version, validated in Brazil $^{(36)}$, which assesses the effect of body weight on quality of life. This instrument contains 31 items distributed in five domains (physical function, selfesteem, sexual life, difficulties in public places and work). Each domain, as well as the total score, has a value from zero (worst quality of life) to 100 (best quality of life).

3) Body satisfaction: the participant will classify her current body silhouette, the silhouette she would like to have and the silhouette she considers healthy / ideal, on a scale of 1 (extremely thin) to 9 (extremely obese), by the figure rating scale of Stunkard ${ }^{(37)}$ and Pulvers ${ }^{(38)}$.

4) Degree of satisfaction with the intervention (exercise or microcurrent): the participant will answer eight questions regarding her satisfaction with the intervention, on a scale from 0 (no satisfaction) to 5 (maximum satisfaction), as proposed by Fornell et al. ${ }^{(39)}$ and adapted by the researchers for this study.

5) Assessment of the lumbopelvic complex: includes the assessment of four muscle groups (1) FTflexors of trunk, (2) ET-extensors of trunk, (3) FL-flexors of lateral trunk and (4) FET-associated flexors and extensors of trunk, as proposed by Penã et al. ${ }^{(40)}$.

\section{Statistical analysis}

The statistical analysis will be performed in the Statistical Package for The Social Sciences (v. 22, SPSS, Inc., IL, USA) software by a researcher blinded to the allocation of participants to groups. The data will be submitted to the Kolmogorov-Smirnov test to test normality of distribution and Levene's test for homogeneity.

Parametric data will be expressed as mean and standard deviation, while non-parametric data will be presented as median, minimum and maximum. The Mauchly test will be used to verify the specificity of the data and, if not validated, the effects and interactions will be analyzed using the mixed model ANOVA test, with confidence intervals being presented.

The comparison between the groups in the pre, after 6 weeks, and at the end of the intervention will be carried out by the mixed model ANOVA, and the Bonferroni post-hoc will be used to verify the time factor (pre, 6 weeks and post) and groups (CG, EG and MEG), and the interaction between the factors.

The magnitude of the difference between the interventions will be given by the effect size analysis. For numerical variables, intragroup differences before and after will be measured using the paired Student's $t$ test.
For categorical variables, intra-group differences before and after will be measured using the Chi-square test. The level of significance adopted will be $95 \%$ ( $p$ $<0.05$ ). The intraclass correlation coefficient (ICC) and the standard error of measurement (SEM) will be calculated for body composition/adipometry and anthropometric measurements.

The calculation of the minimum detectable change (MDC), used to verify the notable change in skill in relation to a measure, will be calculated by the equation $M D C=1.96 * \sqrt{ }\left(2^{*} S E M\right)$, in which SEM = standard deviation * $\sqrt{ }(1-I C C)$.

\section{DISCUSSION}

This study aims to verify the effects of high-intensity interval training (HIIT), associated or not with previous application of microcurrent therapy, in reducing abdominal fat in sedentary women, aged 18 to 40 years. Recent systematic reviews with meta-analysis ${ }^{(10,41)}$ proved that HIIT and continuous training of moderate intensity similarly promote reductions in body mass, fat mass and risk indicators for cardiovascular and metabolic diseases in overweight or obese adults.

However, it appears that HIIT promotes greater reduction in total absolute fat mass ${ }^{(10)}$, spending less practice time $^{(11)}$ and providing high adherence rates ${ }^{(8)}$. This type of training acts directly on the metabolism of fats, causing better use of the energy produced by the breakdown of adipocytes, making consumption more effective $^{(42)}$. On the other hand, it is also suggested that the therapeutic application of low frequency electrical current acts directly on the accumulated adipocytes, causing their local destruction (electrolipolysis) and favoring their subsequent elimination ${ }^{(43)}$. Initially, the technique was applied with the use of needle electrodes in the adipose panicle in milliamperage, however evidence has shown that the use of electrodes in a transcutaneous way shows similar results in abdominal perimetry $^{(16)}$, even without associating the practice of exercise. Interestingly, another study found that the results obtained with the use of needles were more expressive for sedentary women than active women ${ }^{(17)}$.

Following the logic that cell reactions are mediated in part by electrical signals in the microampere range ${ }^{(19)}$, it is believed that the microcurrent emitted in microamperage can favor and accelerate the reduction of localized adiposity. However, the exact mechanisms by which electric fields can reduce localized adiposity are still not fully understood. Poltawski \& Watson ${ }^{(44)}$ report that the microcurrent increases the number of organelles responsible for cellular activities and the concentrations of adenosine triphosphate that provides cellular energy, favoring metabolic exchanges, amino acid transport and protein synthesis ${ }^{(2)}$.

It is suggested that the microcurrent stimulates sympathetic nervous excitations with the release of catecholamines (adrenaline and norepinephrine) that act on the adipocyte receptors and stimulate the increase of the cAMP enzyme, increasing the activation of protein kinase $A$ and lipolytic enzymes (sensitive lipase hormone and lipoprotein lipase), causing 
hydrolysis of triglycerides to glycerol and fatty acids, which translates into lipolysis and increased local catabolism. After the fatty acid is combined with albumin to create a free fatty acid that can be used as an energy source during exercise ${ }^{(2,21)}$. Thus, when its application is associated with the practice of physical exercise, there is an increase in urine glycerol with a higher lipolytic rate compared to exercise alone ${ }^{(5)}$.

Thus, a previous study ${ }^{(2)}$ which aimed to analyze the effects of microcurrent on abdominal fat in a transcutaneous or percutaneous form applied before or after aerobic exercise, proved that the previous application of transcutaneous form was more effective suggesting that the greater contact area with the abdominal region may have provided ample stimulation of $b$-adrenergic receptors. Furthermore, to achieve these therapeutic benefits, dosimetry of the resource becomes a crucial point and involves regulating the following parameters: stimulation frequency, current amplitude, electrode position, duration of application, number of applications per day and number of days a week $^{(19)}$

The stimulation frequency generally ranges from 5 to $50 \mathrm{~Hz}^{(45)}$, wherein a previous study ${ }^{(21)}$ demonstrated that frequencies between 10 to $25 \mathrm{~Hz}$ (compared to $50 \mathrm{~Hz}$ ) showed a better reduction in visceral and abdominal fat, and increased lean mass and triglyceride levels in the bloodstream. Thus, it is suggested to apply $25 \mathrm{~Hz}$ in the first 15 minutes and $10 \mathrm{~Hz}$ in the last 15 minutes to avoid desensitization of adipocytes ${ }^{(2)}$ enhancing the stimulation of lipolytic mediators released by the adrenergic sympathetic system ${ }^{(5)}$.

It is believed that different amplitudes can be used, as long as they do not exceed $1000 \mu \mathrm{A}$ and are in a tolerable range for the patient at the wound site ${ }^{(19)}$. Cheng et al. ${ }^{\left({ }^{(4)}\right)}$ demonstrated that the therapeutic range is located between 10 to $1000 \mu \mathrm{A}$ for the skin of rats, with the greatest increase in the incorporation of glycine and aminoisobutyric acid in the skin protein, in relation to the control group, occurred in the intensity of $500 \mu \mathrm{A}(123 \%$ and $90 \%$ increase, respectively).

Whereas the amino acid transport increased from 30 to $40 \%$ in intensity between 100 and $500 \mu \mathrm{A}$ compared to the control group and the increase in protein synthesis can be partially answered by the increase in ATP production. As for the time and frequency of application, there is a lack of evidence to determine the selection.

It is suggested that the application can vary from 30 to 120 minutes, 1 to 4 applications a day for 5 to 7 days a week ${ }^{(19)}$, wherein times over 30 to 40 minutes $^{(45)}$ appear to be more effective. Therefore, the microcurrent dosimetry of our study was based on the three protocols applied by this group of researchers ${ }^{(2,5,21)}$. It can be seen, then, that both the microcurrent and HIIT have scientific proof of their effectiveness in reducing abdominal fat, but there is still no evidence in the literature of the application of microcurrent associated with HIIT exercises. Considering this, it is suggested that further studies could be carried out combining both interventions, looking for an effective alternative for the reduction of abdominal fat in the population in question. Therefore, this study defined a specific intervention protocol for the association of the techniques, in order to prove its effectiveness.

The present clinical trial may have a limitation due to the impossibility of monitoring the participants' caloric intake; there is no more detailed and specific assessment of abdominal fat; difficulties for participation and commitment of the participants to spend the time necessary for interventions and evaluations. In addition, the protocol does not include ultrasound to measure the thickness of the abdominal fat layer and a photographic record of the abdominal region to interpret the image in software in order to analyze abdominal fat in detail.

Author's contribution: RIGK defined the concept of the study, created the hypothesis and wrote the original proposal. ASV, ACBM and RBSG were involved in the critical review of the study proposal and the manuscript. AP, NLS, NPS, RASA wrote this study protocol, with the contribution of all co-authors. All authors read and approved the manuscript.

Financial support: This study will be partially funded in the form of a Scientific Initiation Scholarship, granted to the student, by the Federal University of Paraná. The corresponding author will be the researcher-sponsor of this study and will manage the study design, collections, data analysis and interpretation, the writing of the manuscript and the decision to submit for publication.

Conflict of interest: The authors declare that they have no conflict of interest.

\section{REFERENCES}

1. Silva RMV, Silva MWT, Medeiros SF, Guerra SSB, Nobre RS, Meyer PF. Effects of cryofrequency on localized adiposity in flanks. Man Ther Posturology Rehabilt J 2019;16:551.

2. Noites A, Pinto J, Freitas CP, Melo C, Albuquerque $A$, Teixeira $M$ et al. Effects of microcurrent and physical exercise on the abdominal fat in patietns with coronary artery disease. Eur J Integr Med 2015;7(5):499-507.

3. Kofler T, Bossard M, Aeschbacher S, Tabord A, Duncan DI, Kim TH, et al. A prospective study analyzing the application of radiofrequency energy and high-voltage, ultrashort pulse duration electrical fields on the quantitative reduction of adipose tissue. J Cosmet Laser Ther.2016;18(5):257-67.

4. Mello PB, Dreher PM, Piccinini AM, Rosa LHT, Rosa PV. Comparação dos efeitos da eletrolipólise transcutânea e percutânea sobre a gordura localizada na região abdominal e de flancos através da perimetria e análise de bioimpedância elétrica. Fisioterapia Brasil. 2010;11(3):198-203.

5. Melo NR, Monteiro FMAC, Pontes GAR, Mello SMB. Eletrolipólise por meio da estimulação nervosa elétrica transcutânea (Tens) na região abdominal em pacientes sedentárias e ativas. Fisioter Mov. 2012;25(1):127-40.

6. Balakatounis KC, Angoules AG. Low-intensity electrical stimulation in wound healing: review of the efficacy of eternally applied currents resembling the current of injury. Eplasty 2008;8:283-91. 
7. Bélanger AY. Therapeutic electrophysical agents: evidence behing practice. 3 ed. Baltimore: Lippincott Williams \& Williams, 2015.

8. Melo ASC, Moreira JS, Noites A, Couto MF, Melo CA. Clay body wrap with microcurrent: effects in central adiposity. Applied Clay Science. 2013;8081:140-6.

9. Noites A, Nunes R, Gouveia Al, Mota A, Viera Á, Adubeiro $\mathrm{N}$ et al. Effects of aerobic exercise associated with abdominal microcurrent: a preliminar study. J Altern Complement Med. 2015;21(4):229-36.

10. Schulz KF, Altman DG, Moher D, CONSORT Group. CON- SORT 2010 statement: updated guidelines for report- ing parallel group randomized trials. Ann Intern Med. 2010;152(11):726-32.

11. Niwa ABM, Shono M, Mônaco P, Prado G, Osório N. Experiência no uso do ultrassom focado no tratamento da gordura localizada em 120 pacientes. Surg Cosmet Dermatol. 2010;2(4):3235.

12. Kofler T, Bossard M, Aeschbacher S, Tabord A, Repilado FJR, Lely S, et al. The interrelationships of birthweight, inflammation and body composition in healthy adults. Eur J Clin Invest. 2016;46(4):3428.

13. Meadows R, Bower JK. Associations of anthropometric measures of obesity with physical limitations in older adults. Disabil Rehabil.2020;42(8):1101-6.

14. Noites A, Moreira A, Melo C, Faria M, Vilarinho R, Freitas $C$, et al. Acute effects of physical exercise with microcurrent in the adipose tissue of the abdominal region: a randomized controlled trial. Eur J Integr Med 2017;9:79-85.

15. Batrakoulis A, Fatouros IG, Chatzinikolaou A, Draganidis D, Georgakouli K, Papanikolaou K, et al. Dose-response effects os high-intensity interval neuromuscular exercise training on weight loss, performance, health and quality of life in inactive obese adults: study rationale, design and methodos of the DolT tiral. Cont Clinical Trials. 2019;15:100386.

16. American College of Sports Medicine (ACSM). ACSM's Guidelines for exercise testing and prescription. 10 ed. Philadelphia: Wolters Kluwer/Lippincott Williams \& Wilkins Health, 2018.

17. Vella CA, Taylor K, Drummer D. High-intensity interval and moderate-intensity continuous training elicit similar enjoyment and adherence levels in overweight and obese adults. Eur J Sport Sci. 2017;17(9):1203-11.

18. Maclnnis MJ, Gibala MJ. Physiological adaptations to interval training and the role of exercise intensity. J Physiol. 2017;595(9):2915-30.

19. Viana RB, Naves JP, Coswig VS, Lira CAB, Steele $J$, Fisher JP, et al. Is interval training the magic bullet for fat loss? A systematic review and metaanalysis comparing moderate-intensity continuous training with high-intensity interval training. $\mathrm{Br} \mathrm{J}$ Sports Med. 2019;53(10):655-64.
20. Zhang H, Tong TK, Qiu W, Zhang X, Zhou S, Liu Y, et al. Comparable effects of High-Intensity Interval Training and prolonged continuous exercise training on abdominal visceral fat reduction in obese young women. J Diabetes Res. 2017;2017:5071740.

21. Alizadeh Z, Halabchi F, Mazaheri R, Abolhasani M, Tabesh M. Review of the mechanisms and effects of noninvasive body contouring devices on cellulite and subcutaneous fat. Int $\mathrm{J}$ Endocrinol Metab. 2016;14(14):e36727.

22. Friedman DP. A review of the aesthetic treatment of abdominal subcutaneous adipose tissue: background, implications, and therapeutic options. Dermatol Surg. 2015;41(1):18-34.

23. Mazzoni D, Lin MJ, Dubin DP, Khorasani H. Review of non-invasive body contouring devices for fat reduction, skin tightening and muscle definition. Australas J Demartol. 2019;60(4):278-83.

24. Jackson AS, Pollok ML. Practical assessment of body composition. Phys Sportsmed. 1985;13(5):7690.

25. Hallal PC, Andersen LB, Bull FC, Guthold R, Haskell W, Ekelund U. Global physical activity levels: surveillance progress, pitfalls, and prospects. The Lancet. 2012; 380(9838):247-57.

26. Ferreira JC, Patino CM. Randomization: beyond tossing a coin. J. bras. pneumol. 2016;42(5):310310.

27. Roy BA. High-Intensity Interval Training: eficiente, effective, and a fun way to exercise: brought to you by the American College os Sports Medicin. ACSM's Health \& Fitness Journal. 2013;17(3):3.

28. Gibala MJ, Little JP, Macdonald MJ, Hawley JA. Physiological adaptations to low-volume, highintensity interval training in health and disease. $J$ Physiol. 2012;590(5):1077-84.

29. Borg C. Ratings of perceived exertion and heart rates during short-term cycle exercise and their use in a new cycling strength test. Int $\mathrm{J}$ Sports Med. 1982;3(3):153-8.

30. Tanaka H, Monahan KD, Seals DR. Age-predicted maximal heart rate revisited. J Am Coll Cardiol. 2001;37(1):153-6.

31. Cômodo ARO, Dias ACF, Tomas BZ, Silva-Filho AA, Werustsky CA, Ribas DF, et al. Utilização da bioimpedância para avaliação da massa corpórea. Associação Médica Brasileira e Conselho Federal de Medicina: Projeto Diretrizes, 2009.

32. Faulkner JA. Physiology of swimming. Res $Q$. 1996;37(1):41-54.

33. World Health Organization (WHO). Waist circumference and waist-hip ratio: report of a WHO expert soncultation. Geneva: WHO; 2008.

34. Matsudo S, Araújo T, Matsudo V, Andrade D, Andrade E, Oliveira LC, et al. Questionário Internacional de Atividade Física (IPAQ): estudo de validade e reprodutibilidade no Brasil. Rev Bras Ativ Fís Saúde. 2011;6(2):5-18.

35. International Physical Activity Questionnaire. Guide- 
lines for data processing and analysis of the international physical activity questionnaire (IPAQ): short and long forms; 2005.

36. Mariano MHA, Kolotkin RL, Petribú K, Ferreira NLM, Dutra RF, Barros MV, et al. Psychometric Evaluation of a brazilian version of the impact of weight on quality of life (IWQOL-Lite) instrument. Eur Eat Disord Rev. 2010;18(1):58-66.

37. Stunkard AJ, Sorensen T, Schulsinger F. Use of the danish adoption register for the study of obesity and thinness. Res Publ Assoc Res Nerv Ment Dis. 1983;60:115-120.

38. Pulvers KM, Lee RE, Kaur H, Mayo MS, Fitzgibbon $M L$, Jeffries SK, et al. Development of a culturally relevant body image instrument among urban African Americans. Obes Res. 2004;12(10):164151.

39. Fornell C, Johnson MD, Anderson EW, Cha J, Bryant BE. The American Customer Satisfaction Index: nature, purpose, and finding. Journal of Marketing. 1996;60(4):7-12.

40. Peña G, Elvar JHR, Moral S, Donate FI, Ordoñez FM. Revisión de los Métodos de valoración de la estabilidad central (Core);2012.

41. Andreato LV, Esteves JV, Coimbra DR, Moraes AJP, Carvlho T. The influence of high-intensity interval training on anthropometric variables of adults with overweight or obesity: a systematic review and network meta-analysis. Obes Rev. 2019;20(1):142-55.

42. Andreato LV, Esteves JV, Coimbra DR, Moraes AJP, Carvlho T. The influence of high-intensity interval training on anthropometric variables of adults with overweight or obesity: a systematic review and network meta-analysis. Obes Rev. 2019;20(1):142-55.

43. Andreato LV, Esteves JV, Coimbra DR, Moraes AJP, Carvlho T. The influence of high-intensity interval training on anthropometric variables of adults with overweight or obesity: a systematic review and network meta-analysis. Obes Rev. 2019;20(1):142-55.

44. Carter H, Pringle JS, Jones AM, Doust JH. Oxygen uptake kinetics during treadmill running across exercise intensity domains. Eur J Appl Physiol. 2002;86(4):347-54.

45. Silva RMV, Basilio FB, Nóbrega MG, Medeiros CBR. Efeitos da eletrolipólise na adiposidade abdominal: revisão. Revista Pesquisa em Fisioterapia. 2016;6(1):65-72.

46. Potawski L, Watson T. Bioelectricity and microcurrent therapy for tissue healing- a narrative review. Phys Ther Rev. 2009;14(2):104-14.

47. Borges FS. Dermato-funcional: modalidades terapêuticas nas disfunções estéticas. São Paulo: Phorte; 2010.

48. Cheng N, Van Hoof H, Bockx E, Hoogmartens MJ, Mulier JC, De Dijcker FJ et al. The effects of electric currents on ATP generation, protein synthesis, and membrane transporto of rat skin. Clin Orthop Relat Res. 1982;(171):264-72.
Appendix 1: Detailed description of the High Intensity Interval Training protocol.

Modality: High-intensity Interval Training. Duration: 30 minutes (5 minutes of warm-up on the exercise bike, 20 minutes of a high-intensity interval training circuit containing 10 sets of 60 seconds interval with 60second active rest and 5 minutes of cool-down). Frequency: 2 times a week, totaling 10 interventions. Intensity: High, above $80 \%$ of $\mathrm{HR}_{\max }$ (monitored by the frequency meter) and Borg Scale above 15 - tiresome (monitored by the participant and researcher in the 12th and 22nd minutes of exercise). Supervision: Physiotherapist Researcher.

Previous guidelines for the participant to perform the intervention: 1) wear comfortable clothes that facilitate the execution of movements; 2) not be fasting;

3 ) having eaten the last meal more than two hours ago;

4) preferably, empty the bladder.

\section{Description of the circuit containing the 10 series of 60 seconds}

Observation: between each series the participant must perform, during 60 seconds, active rest with constant movement of the members, being able to choose: 1) in orthostatic position, keep feet supported and perform knee flexion-extension, alternately, as if you were running in place; 2 ) in orthostatic position, with the feet supported, perform unilateral knee flexion-extension removing the contralateral foot from the support and vice versa; 3) in orthostatic position, keep feet supported and knees extended to perform movements with upper limbs alternating clapping of the hands in front of and behind the body.

Series 1. High knees on a trampoline: in orthostatic position, keep upper limbs with shoulder in neutral position, elbow flexed at $90^{\circ}$ and palms facing down. Remove one foot from the support, performing maximum hip and knee flexion, alternating bilaterally, simulating a run in place.

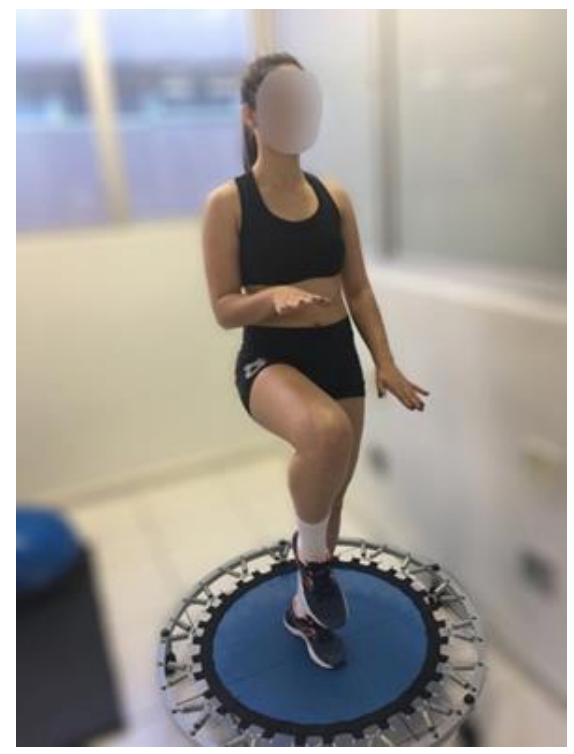


Series 2. BOSU Sit-ups: in supine position, keep the thoracic spine supported on the BOSU remaining with approximately $40^{\circ}$ of trunk flexion; upper limbs with shoulder at $90^{\circ}$ of abduction, elbows at $90^{\circ}$ of flexion, hands closed and lower limbs with hips and semi-flexed knees and feet flat on the floor. Perform unilateral trunk flexion and rotation, extending the contralateral elbow in front of the body. Repeat successively.
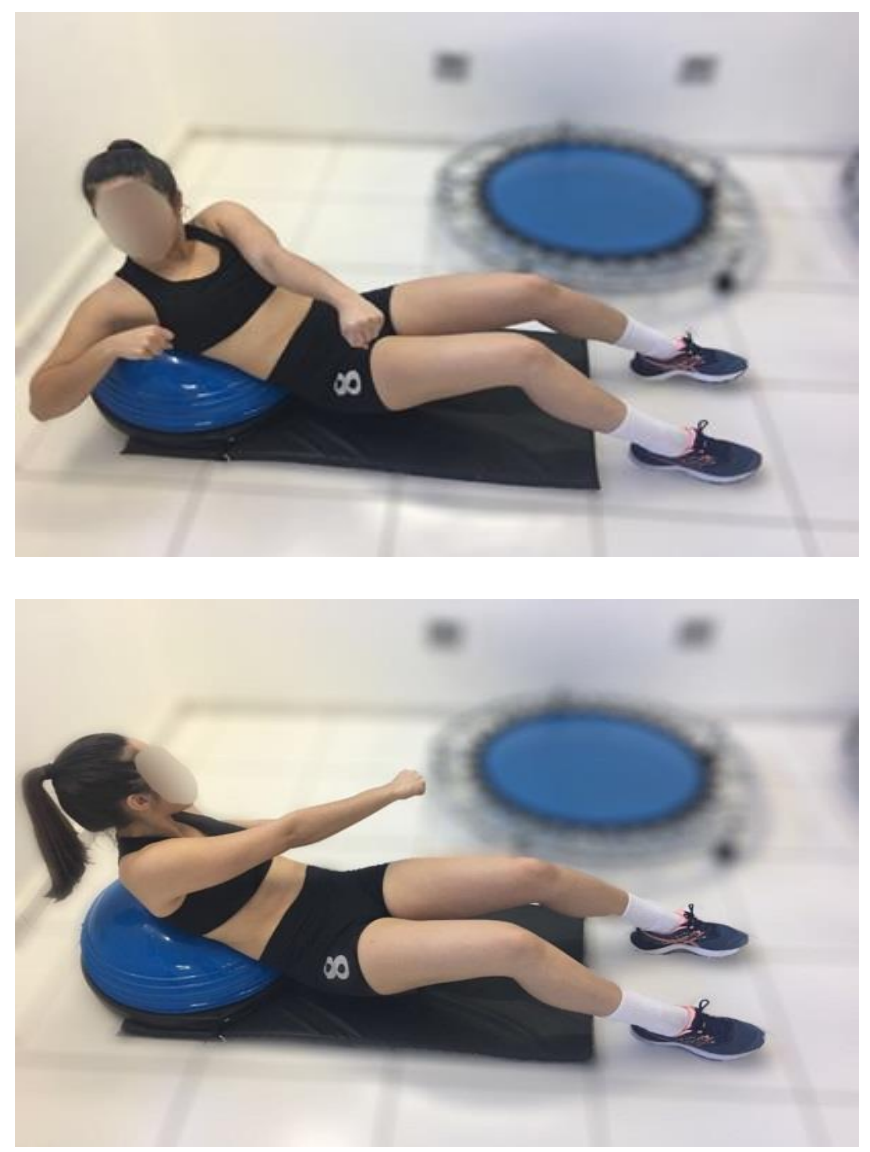

Series 3. Jumping Jack on a trampoline: in orthostatic position, upper limbs in anatomical position and lower limbs with abduction $\left(30^{\circ}\right)$ of hip and extended knee. Perform a small jump, simultaneously with the shoulder abduction movement $\left(180^{\circ}\right)$ and hip adduction. Repeat successively.

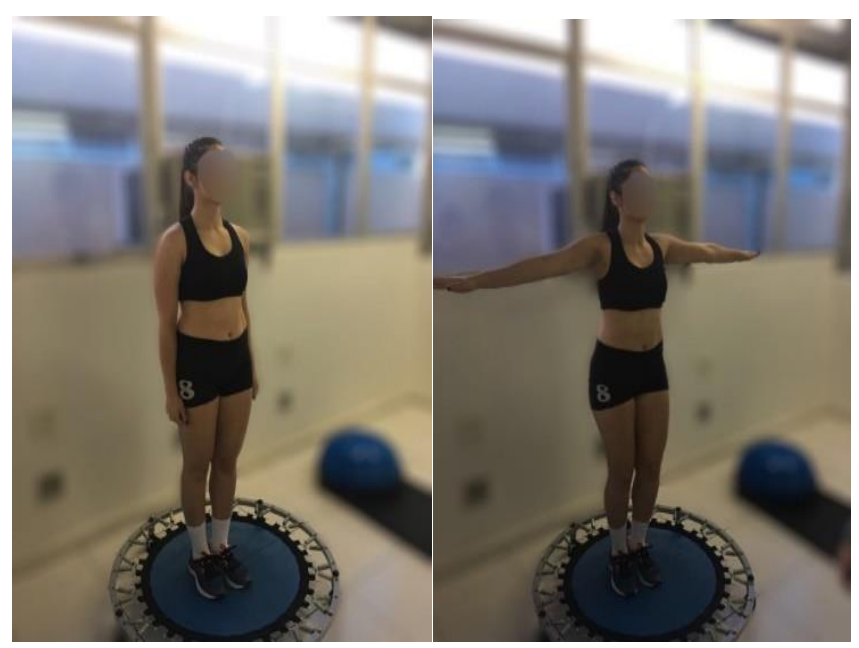

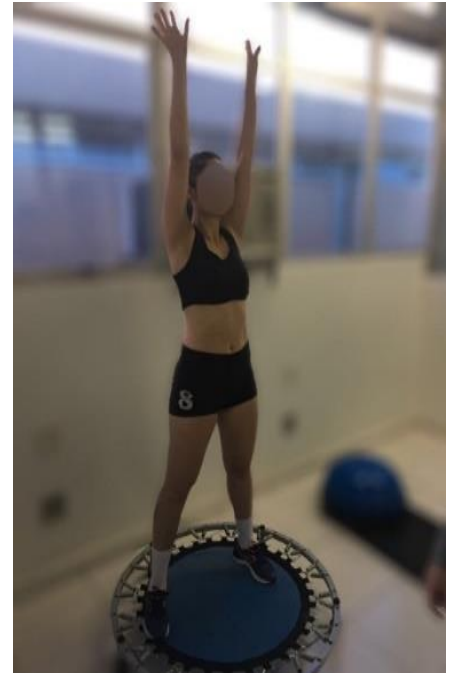

Series 4. Mountain climber: in prone position, remain with the upper limbs with shoulder flexion $\left(90^{\circ}\right)$, elbows extended, wrist extended and palms flat on the floor. Lower limbs with hips in neutral position, knee extended and toes on the floor. Perform the movement of lower limbs (hip and knee flexion) removing the limb from the support alternately, simulating the climb of a mountain.

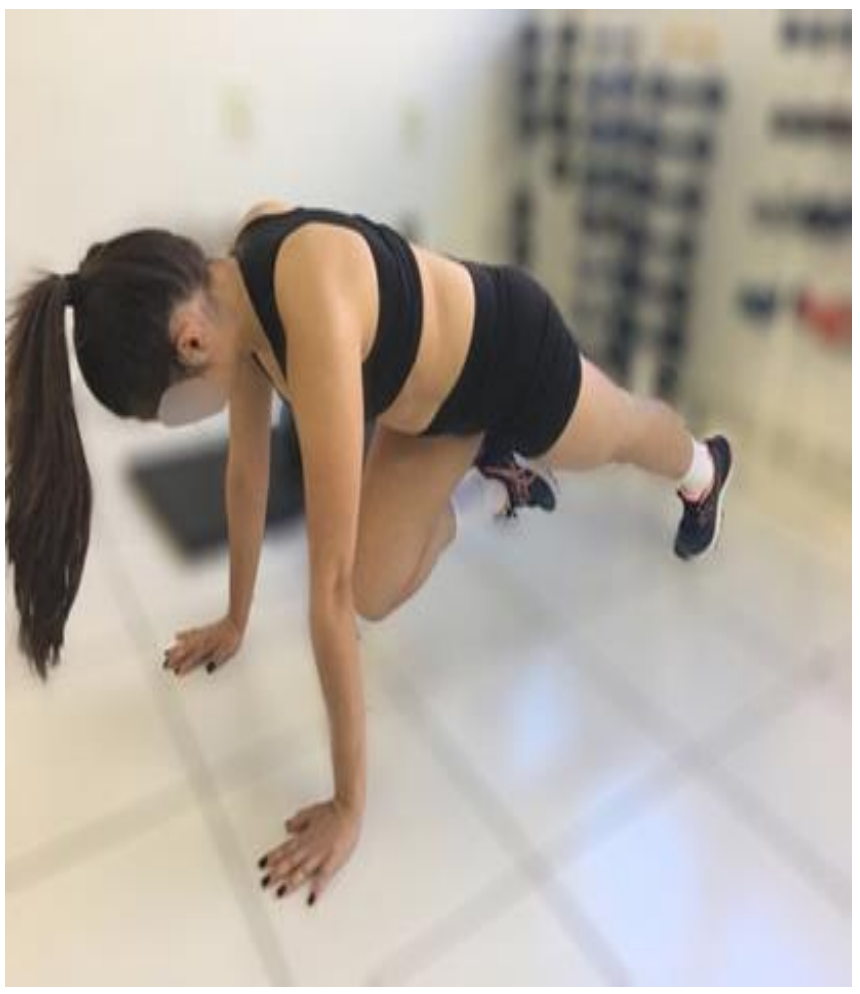

Series 5. Sit-ups with the ball: in supine on a mat, shoulders flexed at $180^{\circ}$ and elbows extended. Place the ball $(60 \mathrm{~cm}$ in diameter) between the malleoli, keeping the lower limbs in light hip flexion and extended knees. Perform trunk and hip flexion movement, transferring the ball to upper limbs and then extending the trunk to the floor. After flexing the torso and hips again, transferring the ball to the lower limbs and returning to the starting position. Repeat this movement successively. 

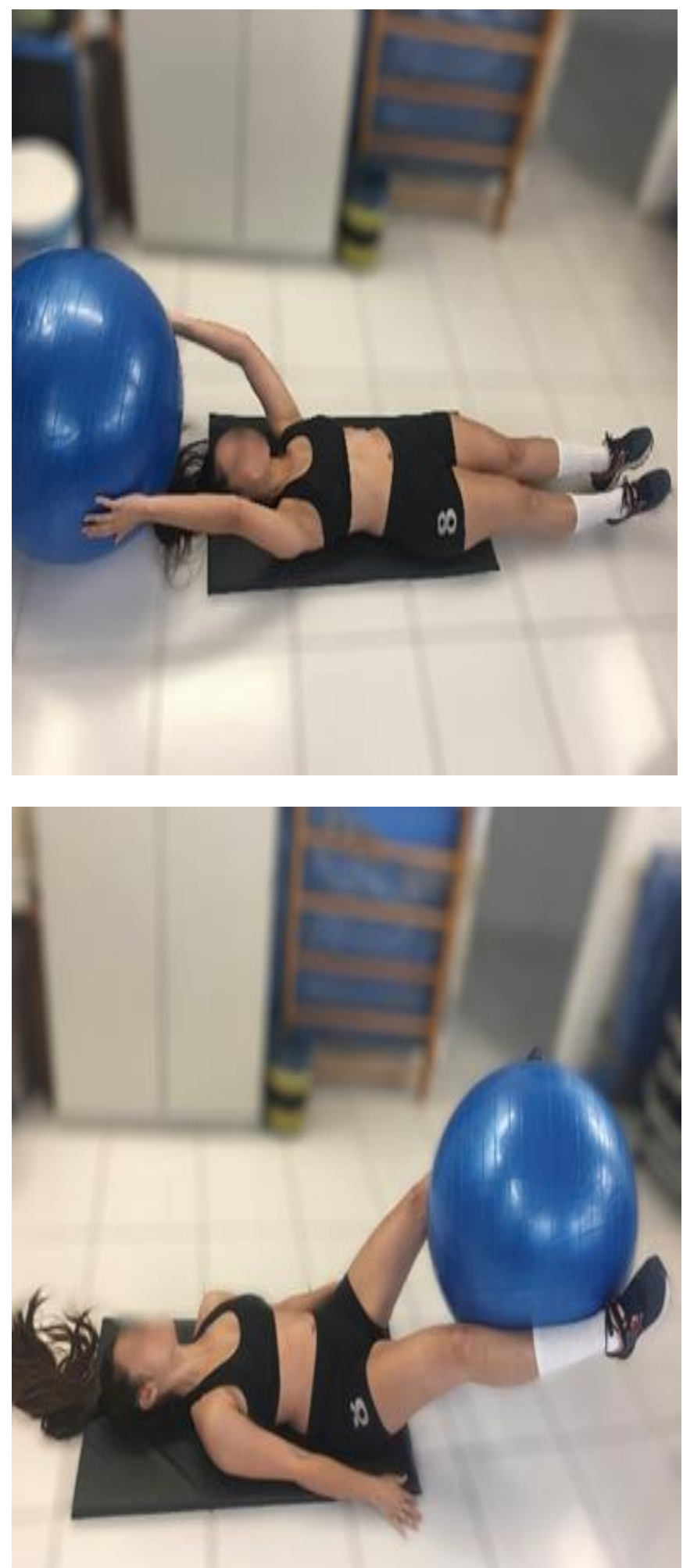

Series 6. Repeat series 1.

Series 7. Repeat series 2.

Series 8. Run with 2 cones positioned $1.5 \mathrm{~m}$ away: Guide the participant to run from one cone to another, bending down and touching the cone. The first cone should be reached with front running and going back to the other cone should be with back running. Repeat successively.

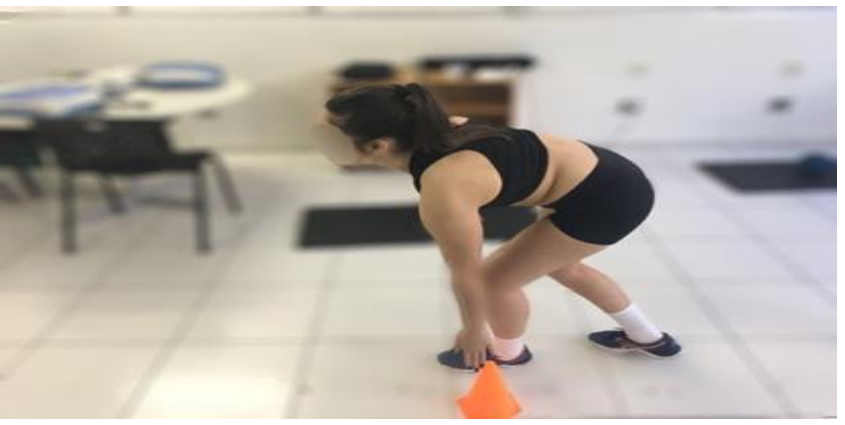

Series 9. Reverse sit-up: in supine on a mat, upper limbs with $90^{\circ}$ abduction, elbows extended and palms facing up. Lower limbs with hips in neutral position and knees extended. Elevate the lower limbs (flexion of the hips and knees simultaneously, until the sacrum is removed from the support) and return to the initial position. Repeat successively.

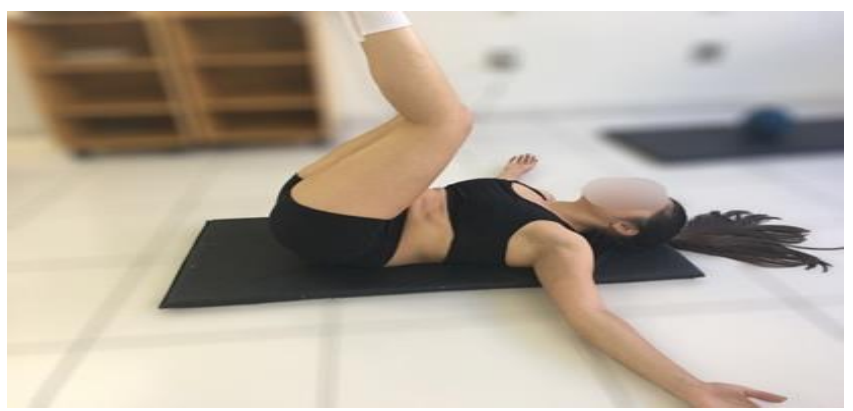

Series 10. Burpee with push-up: Includes three movements. Start in an orthostatic position and then perform a squat with maximum hip and knee flexion supporting your hands on the floor. Then, extend the lower limbs, maintaining the support of the upper limbs, to perform a shoulder and elbow flexion. Return to the crouched position and perform a small jump looking for the orthostatic position while performing shoulder flexion at $180^{\circ}$. Repeat the three movements successively.

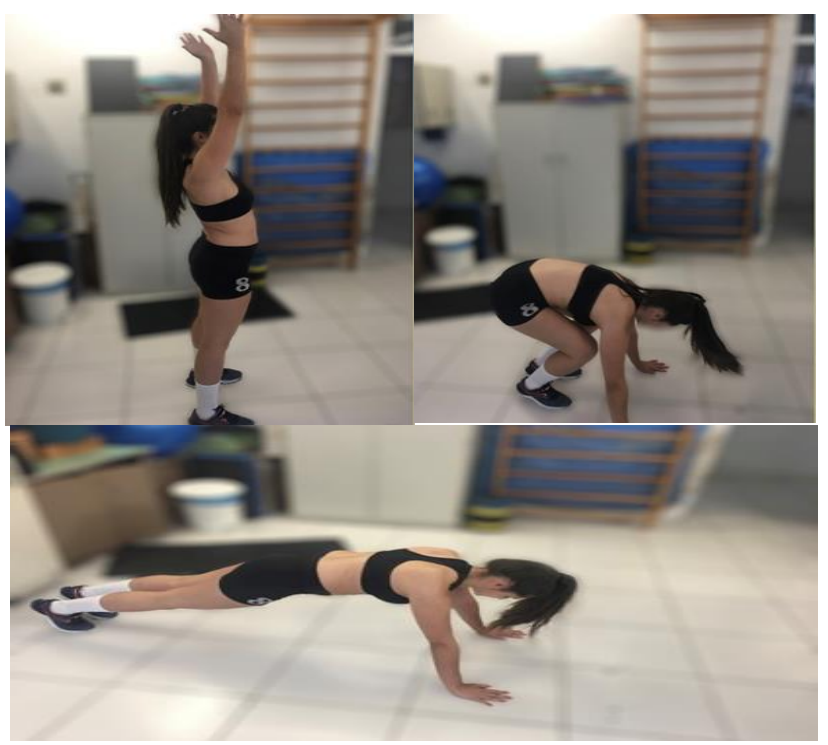

